http://dx.doi.org/10.1590/1678-4162-9036

Arq. Bras. Med. Vet. Zootec., v.69, n.3, p.618-622, 2017

\title{
Eliminação de Dioctophyme renale pela urina em canino com dioctofimatose em rim esquerdo e cavidade abdominal - Primeiro relato no Rio Grande do Sul
}

\author{
[Elimination of Dioctophyme renale to urine in canines with dioctophymosis in the left kidney \\ and abdominal cavity - First report in Rio Grande do Sul] \\ S.C. Perera ${ }^{1}$, J.C.S. Rappeti ${ }^{2}$, V. Milech ${ }^{3}$, F.A. Braga ${ }^{2}$, G.A.O. Cavalcanti ${ }^{2}$,

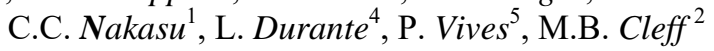 \\ ${ }^{1}$ Programa de pós-graduação - Universidade Federal de Pelotas - Pelotas, RS \\ ${ }^{2}$ Faculdade de Veterinária - Universidade Federal de Pelotas - Pelotas, RS \\ ${ }^{3}$ Programa de pós-graduação - Universidade Federal de Santa Maria - Santa Maria, RS \\ ${ }^{4}$ Médica veterinária autônoma - Palmas, TO \\ ${ }^{5}$ Hospital de Clínicas Veterinária - Universidade Federal de Pelotas - Pelotas, RS
}

\begin{abstract}
RESUMO
Dioctophyme renale é um parasito que afeta tanto animais como humanos e tem como órgão de eleição o rim direito. Relata-se o caso clínico-cirúrgico de um paciente canino com histórico de eliminação de três exemplares de $D$. renale pela urina e presença de parasitos no rim esquerdo e na cavidade abdominal. No Hospital de Clínicas Veterinárias da Universidade Federal de Pelotas, foram realizados exames précirúrgicos, entre eles a ultrassonografia abdominal, que identificou a localização dos nematódeos, e o exame de Doppler pulsado dos vasos intrarrenais, que demonstrou aumento nos índices resistivos nas regiões avaliadas. $\mathrm{O}$ paciente foi conduzido para a realização de laparotomia exploratória, sendo removidos 23 parasitos da cavidade abdominal. A presença de $D$. renale causa graves lesões nos rins e nos demais órgãos da cavidade abdominal, sendo essencial o diagnóstico precoce e a remoção dos nematódeos para recuperação do paciente.
\end{abstract}

Palavras-chave: parasitos, ultrassom abdominal, Doppler pulsado, laparotomia exploratória

\begin{abstract}
Dioctophyme renale is a parasite that attacks animals as well as humans and has the right kidney as a preferred target organ. This document reports the case of a clinical-chirurgical canine patient with a history of elimination of three D. renale samples through its urine and presence of parasites at its left kidney and abdominal cavity. Pre-cirurgical exams were performed at the Veterinary Hospital of the Federal University of Pelotas. Among these exams, abdominal ultrasonography identified the nematode's location and the Pulse-Doppler of the intrarenal vessels showed an increase in the resistive indices of the evaluated areas. An Exploratory Laparotomy was conducted with the patient who had 23 parasites removed from its abdominal cavity. The presence of D. renale causes severe lesions at kidneys and other organs of the abdominal cavity, for this reason early diagnosis and nematode's removal are essential for the patient's recovery.
\end{abstract}

Keywords: parasites, abdominal ultrasonography, Pulse-Doppler, exploratory laparotomy

\section{INTRODUÇÃO}

O nematódeo Dioctophyme renale, conhecido como o "verme gigante do rim", acomete principalmente os cães de vida errante e também o homem (Alves et al., 2007; Nakagawa et al.,
2007). O rim direito é parasitado com maior frequência, ocorrendo a destruição do parênquima do órgão (Urquhart et al., 1998). Porém, esse parasito também pode ser localizado livre na cavidade abdominal e em outros órgãos (Nakagawa et al., 2007).

Recebido em 29 de junho de 2016

Aceito em 07 de julho de 2016

E-mail: soliane.cp@hotmail.com 
Os hospedeiros definitivos eliminam os ovos de Dioctophyme renale pela urina (Urquhart et al., 1998), contaminando o ambiente. Após um período de incubação em meio aquático, os ovos são ingeridos pelo hospedeiro intermediário, $\mathrm{o}$ anelídeo oligoqueta, e se tornam infectantes (Alves et al., 2007). A infecção ocorre pela ingestão de anelídeos aquáticos, assim como de carne crua ou pouco cozida de peixe e rã contendo a larva infectante (Urquhart et al., 1998).

O diagnóstico da dioctofimatose é realizado por meio de achados ocasionais de helmintos em necropsias e procedimentos cirúrgicos (Kommers et al., 1999), exames de ultrassom (Cottar et al., 2012), observação de ovos na urina e de exemplares do nematódeo também na urina ( $\mathrm{Li}$ et al., 2010), sendo esta última pouco relatada na literatura.

Assim, o objetivo deste trabalho é relatar a ocorrência incomum de Dioctophyme renale no rim esquerdo e na cavidade abdominal de uma fêmea canina com eliminação de parasitos pela via urinária.

\section{CASUÍSTICA}

Um paciente da espécie canina, fêmea, sem raça definida (SRD), porte médio, com $12 \mathrm{~kg}$ de peso corporal, adulto, foi atendido no Hospital de Clínicas Veterinária da Universidade Federal de Pelotas (HCV-UFPel). Na anamnese, foi relatado que era um cão errante que havia sido adotado recentemente, e a principal queixa foi de que o paciente havia eliminado três parasitos pela urina com tamanho médio de 15 a $20 \mathrm{~cm}$. No exame clínico geral, observaram-se apenas alterações de condição corporal, classificada como magra, e mucosa oral de coloração rósea pálida.

Foram realizados exames complementares do canino, como hemograma, bioquímica sérica e exame ultrassonográfico abdominal com transdutor linear e frequência de $10 \mathrm{MHz}$. No hemograma e na bioquímica sérica, foram detectadas grave anemia, leucocitose e alterações das enzimas renais, conforme os seguintes resultados: hemácias $3,53 \times 10^{6}$, hemoglobina $7,1 \mathrm{~g} / \mathrm{dL}$, hematócrito $21,4 \%$, leucócitos totais $22.500 / \mu \mathrm{L}, \quad$ segmentados $19.125 / \mu \mathrm{L}$, ureia $133,1 \mathrm{mg} / \mathrm{dL}$, e creatinina $2,4 \mathrm{mg} / \mathrm{dL}$, sendo os valores de referência considerados de acordo com Lopes et al. (2007). Após o diagnóstico de dioctofimatose, o paciente foi internado para estabilização do quadro clínico com fluidoterapia de ringer lactato $\left(10 \mathrm{~mL} \cdot \mathrm{kg}^{-1}\right.$, a cada hora, via IV), cloridrato de tramadol $\left(2 \mathrm{mg} \cdot \mathrm{kg}^{-1}\right.$, BID, via $\mathrm{SC}$ ) e enrofloxacino (5mg. $\left.\mathrm{kg}^{-1}, \mathrm{BID}, \mathrm{VO}\right)$. Após dois dias de internação, o paciente foi encaminhado para a realização de procedimento cirúrgico. Imediatamente antes da cirurgia, realizaram-se exames de Doppler renal e ultrassom abdominal, sendo este último feito para verificar novamente a localização dos exemplares de $D$. renale nos rins.

Como protocolo anestésico, realizou-se medicação pré-anestésica com metadona $\left(0,2 \mathrm{mg} \cdot \mathrm{kg}^{-1}\right.$, via IM), indução anestésica com propofol (3mg. $\mathrm{kg}^{-1}$, via IV) e manutenção com isoflurano ao efeito por via inalatória. No pósoperatório imediato, realizou-se analgesia com meloxicam $\left(0,2 \mathrm{mg} . \mathrm{kg}^{-1}\right.$, via $\left.\mathrm{SC}\right)$ e cloridrato de tramadol (3mg. $\mathrm{kg}^{-1}$, via SC).

Após a indução anestésica e a antissepsia da área cirúrgica, realizou-se celiotomia exploratória, com a remoção de 23 parasitos com tamanho variando entre 15 e $45 \mathrm{~cm}$. Entre os exemplares, 16 eram machos e sete eram fêmeas, dos quais 16 estavam vivos (Fig. 1). A cavidade abdominal foi lavada com solução fisiológica aquecida e, posteriormente, procedeu-se à celiorrafia.

O paciente permaneceu internado por oito dias para administração da fluidoterapia de manutenção, antibioticoterapia e medicação de suporte, mantendo-se o protocolo de analgesia por três dias. Além disso, foi administrado também sulfato ferroso e eritropoetina (100UI.kg-1, a cada 48 horas, via SC), havendo uma elevação progressiva do hematócrito. 


\section{Perera et al.}

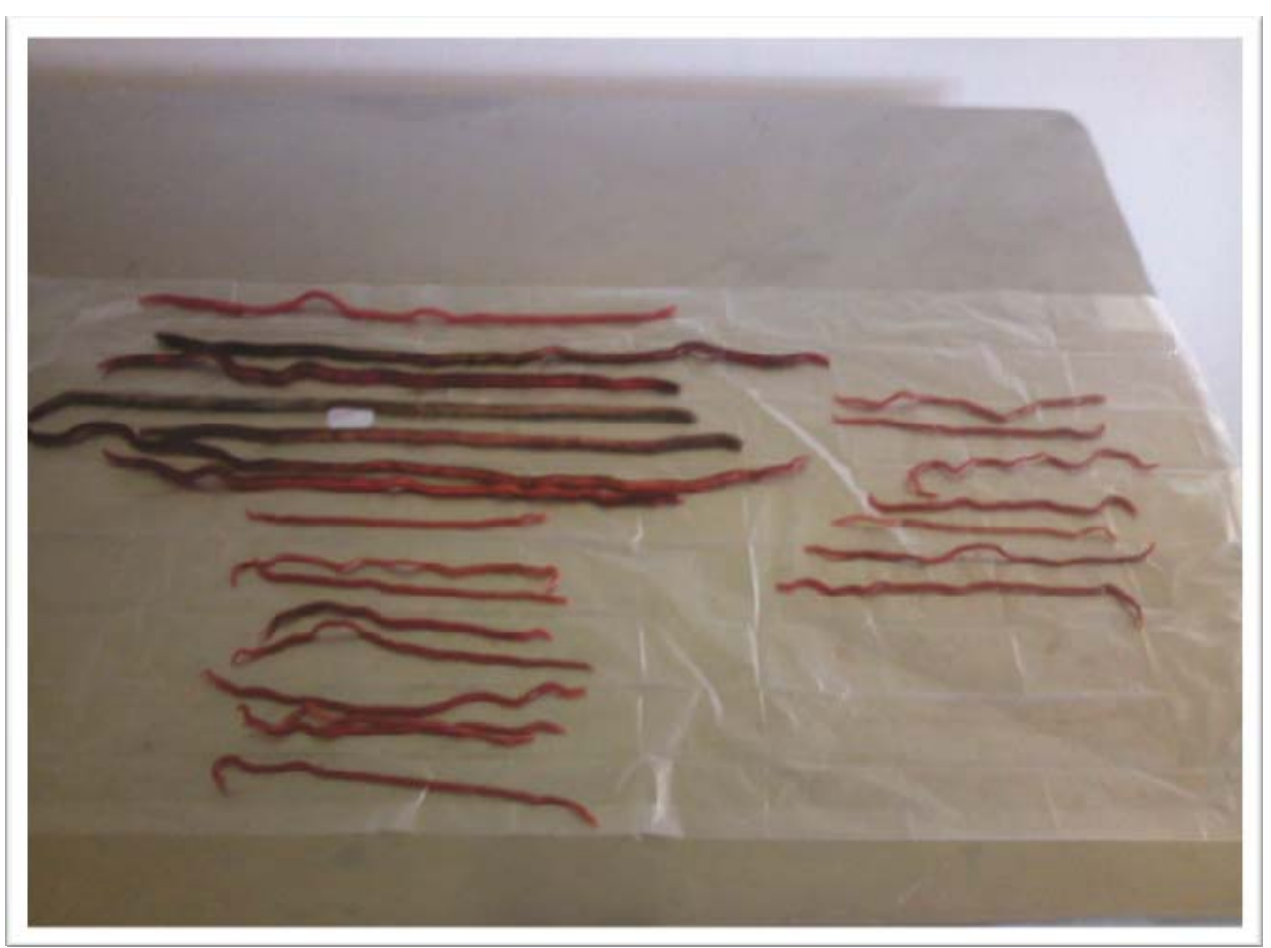

Figura 1. Exemplares de Dioctophyme renale removidos cirurgicamente da cavidade abdominal do paciente canino. Fonte: Arquivo fotográfico do PRODiC.

\section{DISCUSSÃO}

O cão parasitado havia sido recolhido das ruas da cidade, corroborando com Kommers et al. (1999), os quais relatam que a dioctofimatose é uma enfermidade que acomete principalmente animais de vida errante devido a hábitos alimentares pouco seletivos. Ademais, até a eliminação dos parasitos pela urina, os tutores não haviam observado alterações ou sintomas clínicos no canino. Segundo Urquhart et al. (1998), os animais acometidos costumam ser assintomáticos, entretanto, quando há sinais clínicos, estes geralmente estão relacionados à disúria, hematúria e dor lombar.

Uma das formas de diagnóstico da parasitose é a visualização de exemplares de $D$. renale na urina (Li et al., 2010), no entanto isto é raramente encontrado na literatura, sendo este o primeiro relato na veterinária na região do estudo. Stainki et al. (2011) relataram a ocorrência de $D$. renale em um cão de seis meses com obstrução uretral devido à presença do parasito. Por outro lado, em humanos, Li et al. (2010) relataram um caso de dioctofimatose bilateral em uma mulher chinesa, que expeliu fragmentos do parasito pela urina.
Apesar da intensa infestação parasitária, anemia, leucocitose e elevação dos níveis de ureia e creatinina, o paciente canino não apresentava indisposição física. Estes resultados contradizem aqueles observados por Mesquita et al. (2014), que não observaram alterações de ureia e creatinina em nenhum dos oito animais com dioctofimatose renal avaliados précirurgicamente, fato que pode ter ocorrido devido à compensação realizada pelo rim contralateral. Entretanto, Cottar et al. (2012) citam algumas situações em que o rim contralateral não consegue compensar e manter a função renal, o que poderia justificar as alterações observadas nos exames apresentados do paciente aqui relatado.

No primeiro exame de ultrassom do canino, observou-se alteração no baço, com presença de pequenas áreas hipoecogênicas de contorno irregular dispersas pelo parênquima. Ademais, o paciente apresentou a vesícula urinária com grande quantidade de sedimento, omento difusamente hiperecogênico e imagem sugestiva de exemplares de $D$. renale livres na cavidade abdominal. 
Além das alterações citadas, constatou-se que os rins apresentavam formato anatômico alterado: no rim esquerdo, observou-se um aumento de volume e presença de $D$. renale de $0,4 \mathrm{~cm}$ de diâmetro (Fig. 2), enquanto que no rim direito verificou-se diminuição de tamanho, calcificação na região proximal do ureter e sem distinção de cortical e medular. Esta última alteração também foi observada em estudo ultrassonográfico realizado por Mesquita et al. (2014) em cães com dioctofimatose. A presença de calcificação no ureter reforça a hipótese de que o rim direito do paciente pode ter sido parasitado, havendo eliminação do parasito pela via urinária, o que justificaria o relato do proprietário sobre a eliminação dos parasitos.

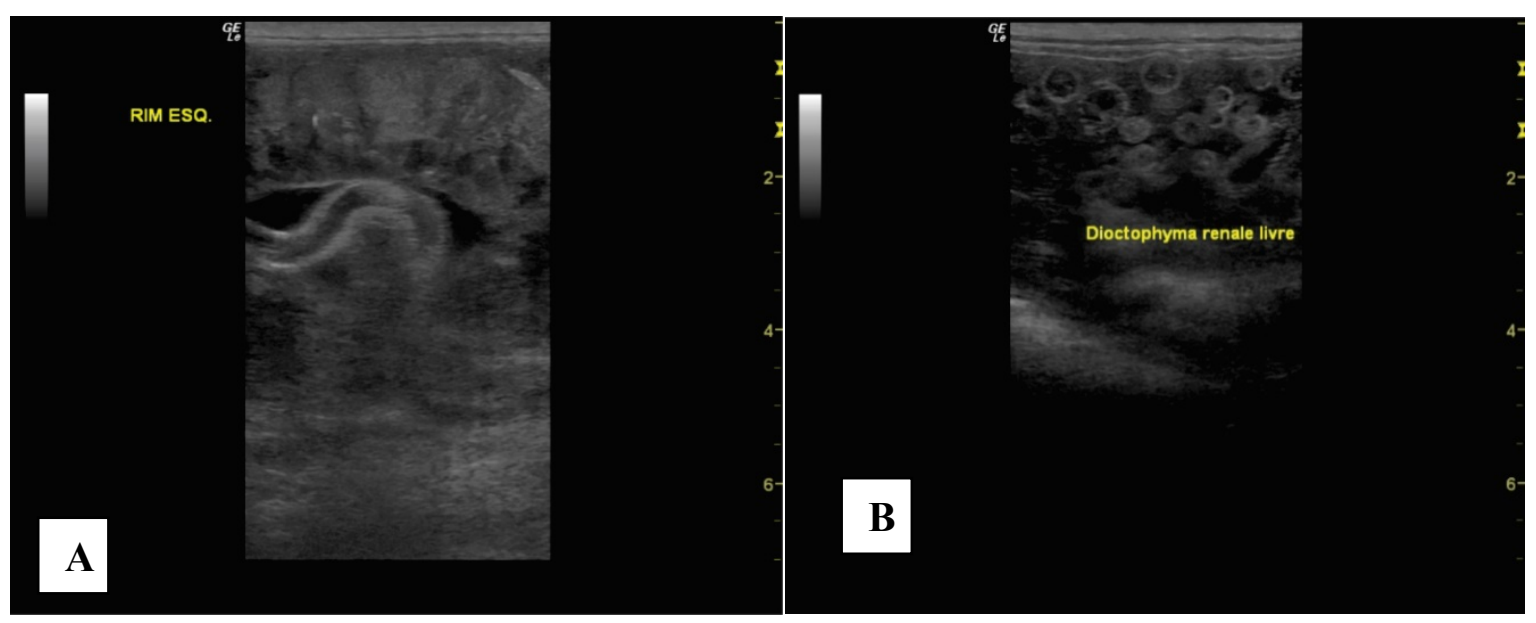

Figura 2. Imagens ultrassonográficas dos exemplares de Dioctophyme renale no interior do rim esquerdo (A) e na cavidade abdominal (B) do paciente canino. Fonte: Arquivo fotográfico do PRODiC.

No segundo exame ultrassonográfico realizado previamente à cirurgia, as alterações observadas inicialmente se mantinham, exceto que não foi observado o parasito no rim esquerdo. Conforme citam Li et al. (2010), isto pode ser explicado pela possibilidade de o paciente ter eliminado o helminto.

A presença de $D$. renale no rim esquerdo é rara (Li et al., 2010), sendo o rim direito o mais acometido (Nakagawa et al., 2007) devido à migração das larvas pela parede duodenal no animal parasitado. No caso de parasitismo na cavidade abdominal e no rim esquerdo, a migração das larvas ocorre pelas curvaturas menor e maior do estômago, respectivamente (Sousa et al., 2011).

No paciente relatado, realizou-se o estudo de Doppler pulsado dos vasos intrarrenais nas regiões cranial, média e distal de ambos os rins. Os índices resistivos (IR) observados nas três regiões avaliadas estavam aumentados, apresentando, respectivamente, os valores de $0,77,0,76$ e 0,74 no rim direito e de $0,73,0,8$ e 0,73 no rim esquerdo (valor de referência
IR $<0,7)$. De acordo com Rivers et al. (1997), o exame de Doppler renal é capaz de caracterizar doenças que alteram o fluxo de sangue nos rins por meio do valor do IR, havendo já relatos de valores de IR aumentados em cães com uropatia obstrutiva, assim como no caso de dioctofimatose relatado no presente trabalho.

Durante o procedimento cirúrgico, observou-se que o omento estava alterado e com coloração avermelhada, enquanto o peritônio estava espessado e com aspecto micronodular. Nesses casos, a peritonite ocorre devido à presença de ovos e à migração dos helmintos na cavidade abdominal, levando a uma reação inflamatória crônica no peritônio, e também devido à presença de ovos eliminados pelos parasitos fêmeas na cavidade abdominal (Pedrassani et al., 2014). Além disso, os rins também apresentavam irregularidades nas cápsulas renais, e os ureteres estavam com espessura aumentada.

No pós-operatório, exames hematológicos e urinálises foram realizados para acompanhamento do quadro clínico do paciente. Nos hemogramas, observou-se acentuada queda 
no hematócrito, agravando o quadro de anemia. $\mathrm{Na}$ avaliação da bioquímica sérica, constatou-se azotemia, enquanto nenhuma alteração foi observada na urinálise. Os resultados semelhantes nos exames de bioquímica sérica antes e após a cirurgia podem ser justificados pela possível presença de parasitos em ambos os rins, o que causou a destruição do parênquima renal (Urquhart et al., 1998; Cottar et al., 2012).

\section{CONCLUSÕES}

De acordo com o apresentado, é possível concluir que a presença de $D$. renale no rim esquerdo e na cavidade abdominal causa danos severos nos órgãos parasitados pelo helminto, principalmente em relação à função renal. Ademais, apesar de rara, a eliminação de parasitos pela urina pode acontecer em infestações parasitárias renais, além de o tratamento cirúrgico ser imprescindível para a remoção dos parasitos e a recuperação do paciente.

\section{REFERÊNCIAS}

ALVES, G.C.; SILVA, D.T.; NEVES, M.F. Dioctophyma renale: o parasita gigante do rim. Rev. Cient. Eletr. Med. Vet., v.4, p1-6, 2007.

COTTAR, B.H.; DITTRICH, G.; FERREIRA, A.A. et al. Achados ultrassonográficos de cães parasitados por Dioctophyma renale: estudo retrospectivo. Vet. Zootec., v.19, p.8-11, 2012.

KOMMERS, G.D.; ILHA, M.R.S.; BARROS, C.S.L. Dioctofimose em cães: 16 casos. Ciênc. Rural, v.29, p.517-522, 1999.

LI, G.; LIU, C.; LI, F. et al. Fatal bilateral dioctophymatosis. J. Parasitol., v.96, p.11521154, 2010.
LOPES, S.T.A.; BIONDO, A.W.; SANTOS, A.P. Manual de patologia clínica veterinária. 3.ed. Santa Maria: UFSM / Departamento de Clínica de Pequenos Animais, 2007. 107p.

MESQUITA, L.R.; RAHAL, S.C.; FARIA, L.G. et al. Pre- and post-operative evaluations of eight dogs following right nephrectomy due to Dioctophyma renale. Vet. Q., v.34, p.167-171, 2014.

NAKAGAWA， T.L.D.R.; BRACARENSE, A.P.F.R.L.; REIS, A.C.F. et al. Giant kidney worm (Dioctophyma renale) infections in dogs from northern Paraná, Brazil. Vet. Parasitol., v.145, p.366-370, 2007.

PEDRASSANI, D.; WENDT, H.; RENNAU, E.A. et al. Dioctophyme renale Goeze, 1982 in a cat with a supernumerary kidney. Braz. J. Vet. Parasitol., v.23, p.109-117, 2014.

RIVERS, B.J.; WALTER, P.A.; POLZIN, D.J.; KING, V.L. Duplex Doppler estimation of intrarenal pourcelot resistive index in dogs and cats with renal disease. J. Vet. Intern. Med., v.11, p.250-260, 1997.

SOUSA, A.A.R.; SOUSA, A.A.S.; COELHO, M.C.O.C. et al. Dioctofimose em cães. Acta Sci. Vet., v.39, 2011.

STAINKI, D.R.; PEDROZO, J.C.S.R.; GASPAR, L.F.J. et al. Urethral obstruction by Dioctophyma renale in puppy. Comp. Clin. Pathol., v.20, p.535-537, 2011.

URQUHART, G.M.; ARMOUR, J.; DUNCAN, J.L.; JENNINGS, A.M. Parasitologia veterinária. 2.ed. Rio de Janeiro: Guanabara Koogan, 1998. $285 \mathrm{p}$. 